\title{
Urinary Ascites: Cryptic Presentation of Unrecognized Urinary Bladder Injury
}

\author{
ANANNA MA ${ }^{\mathrm{a}}$, AHMED MA ${ }^{\mathrm{b}}$, HAQUE WMM ${ }^{\mathrm{a}}$, IQBAL $\mathrm{S}^{\mathrm{a}}$, AHMED $\mathrm{Z}^{\mathrm{a}}$
}

\begin{abstract}
A case of urinary ascites in a 37-year-old woman caused by an unrecognized bladder injury inflicted during hysterectomy done seven months earlier is presented here.
\end{abstract}

The site of injury was located by conventional retrograde cystography and repaired by laparotomy.

The medical record suggesting uneventful peri and postoperative period, absence of expected typical changes

\section{Introduction}

Urinary ascites results from accumulation of urine as free fluid in the peritoneal cavity. In neonates this is usually caused by congenital obstructive uropathy leading to perinephric extravasation of urine or a vesicoperitoneal or ureteroperitoneal fistula formation ${ }^{1-3}$.

Acquired cases that occur in later life are usually caused by an inadvertent bladder or ureteric injury inflicted during a pelvic surgery ${ }^{4-6}$. Injury to urinary bladder is a recognized complication during hysterectomy, other gynecological procedures and laparoscopic surgery ${ }^{7-10}$.

A case of urinary ascites in a 37-year-old woman caused by an unrecognized bladder injury inflicted during a past hysterectomy is reported in this communication.

\section{Case Report}

A 37 yrs old service holder lady was admitted in BIRDEM under surgery unit with gradually increasing generalized dull aching abdominal pain with dysuria. She also developed gradual distension of abdomen followed by scanty micturition. A Computed Tomography scan (CT scan) of abdomen was done which showed presence of moderate ascites (Fig.-1). She was diagnosed as a case of acute abdomen with

a. Dr. Mehruba Alam Ananna, Dr. M Ashrafuddin Ahmed, Dr. WMM Haque, Dr. Sarwar Iqbal, Dr. Ziauddin Ahmed, Department of Nephrology, BIRDEM, Dhaka

b. Dr. M Ashrafuddin Ahmed, Emergency Medical Officer, BIRDEM, Dhaka

Address of correspondence : Dr Mehruba Alam ananna, Registrar, Department of Nephrogy, Room no 1535, BIRDEM , Email : ananna0701@gmail.com in serum biochemistry and the unusual aetilogy of the ascites led to the delay in the diagnosis.

The case demonstrates the importance of high index of suspicion of bladder injury following pelvic surgery in patients with ill-defined symptoms and unexplained ascites even in the absence of typical biochemical changes.

Keywords: Bladder injury, hysterectomy, urinary ascites

(Birdem Med J 2012; 2(1): 60-62)

UTI on the basis of raised ESR, leukocytosis and presence of pus cell in urine and was treated conservatively. The patient was discharged on request but increasing abdominal distention, pain and scanty urine output led to readmission in nephrology department of BIRDEM four days after self discharge.

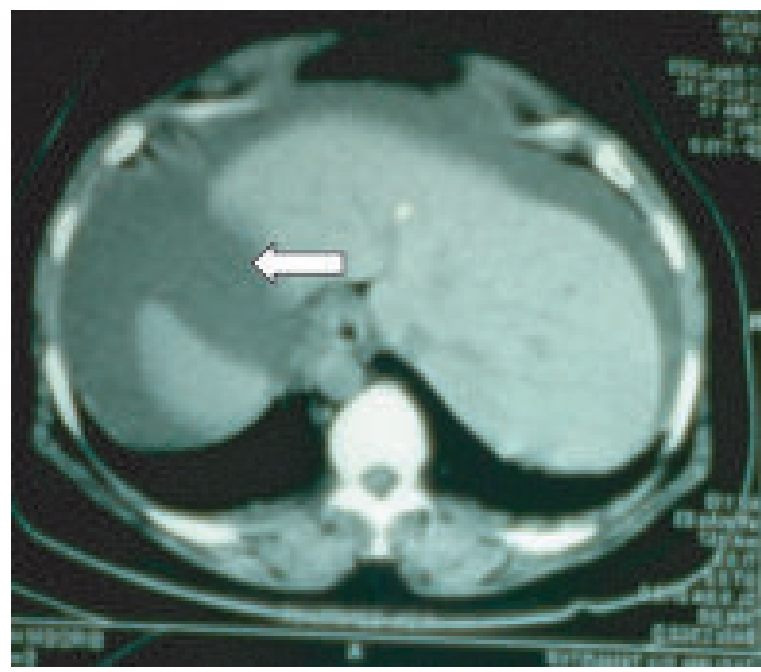

Fig-1 : CT scan of abdomen showing urinary ascites

At the time of admission in our unit she had mild paedal oedma and gross ascites in addition to an old scar mark in her lower abdomen due to abdominal hysterectomy which was performed 7 months back. Other clinical parameters including blood pressure (BP) were normal and there was no proteinuria in bed side urine examination. Abdominal paracentesis was done 3 times both for diagnostic and therapeutic purposes 
and about 3 litres of fluid was aspirated each time with rapid re-accumulation. At the same time she became almost anuric.

During this admission investigation reports showed normal hemoglobin with normal cell count, urine examination was marked by the presence of pus cells (10-15/ hpf) without any haematuria, proteinuria or cast, renal function- serum creatinine- $5.3 \mathrm{mg} / \mathrm{dl}$, blood urea $-140 \mathrm{mg} / \mathrm{dl}, \mathrm{CCr} 40 \mathrm{ml} / \mathrm{hour}$, UTP $0.18 \mathrm{gm} / \mathrm{L}, 24$ hours UTV $200 \mathrm{ml}$. Her serum electrolytes, blood sugar, serum calcium and phoshphate, liver fuction tests, ANA, serum CA-125, serum lipase and amylase all were within normal range. Hepatitis viral markers were negative. USG of whole abdomen confirmed the presence of huge ascites with thickened gall bladder and cystitis. Ascitic fluid study revealed exudative type of fluid with normal cell count and no AFB or malignant cells. She was diagnosed as a case of acute kidney injury (AKI) caused by NSAID which she took for her mechanical pain and was treated conservatively.

Despite improvement of her renal function after stoppage of NSAID, she remained oliguric and ascitic fluid reaccumulated even after repeated aspirations. At this point ,biochemical examination of ascitic fluid revealed very high urea and creatinine $(158 \mathrm{mg} / \mathrm{dl}$ and $18.9 \mathrm{mg} / \mathrm{dl}$ respectively) while her serum urea and creatinine were markedly lower $(16 \mathrm{mg} / \mathrm{dl}$ and 1.96 $\mathrm{mg} / \mathrm{dl}$ respectively). Following per urethral catheterization, six litres urine came out resulting in significant reduction of abdominal distension but the patient developed hypotension, perhaps due to this rapid decompression and was transferred to Intensive Care Unit. After improvement of general condition she was transferred to ward where a retrograde cystogram was done which revealed lack of adequate distension of the urinary bladder due to prompt leakage of contrast material through its right postero-lateral wall into peritoneal cavity (Fig.-2). Depending on these investigations, she was was diagnosed as a case vesicoperitoneal fistula with resolved AKI (NSAID induced) and she was referred to urology department where laparotomy with repair of urinary bladder .

After surgery, patient became symptom free, her renal function became normal and USG of abdomen showed no fluid collection in peritoneal cavity. A repeat cystogram two months later revealed an adequately distended urinary bladder with no sign of any contrast leakage into the peritoneal cavity (Fig.-3 ).

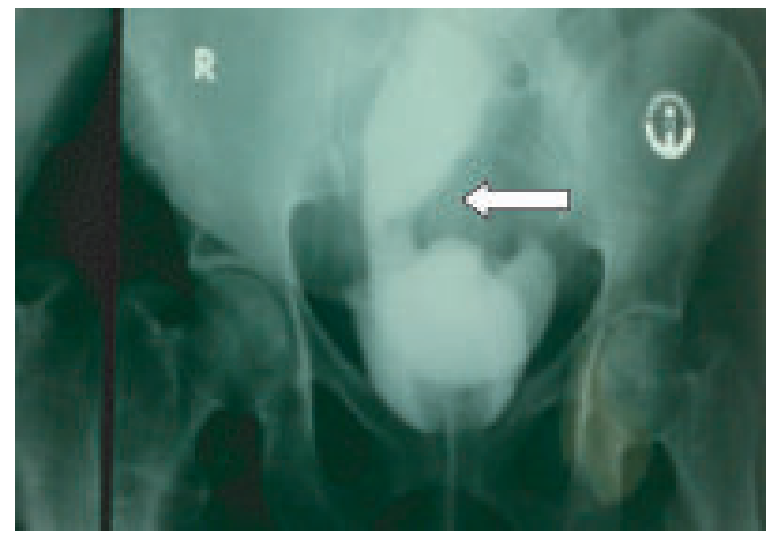

Fig-2: Cystogram showing leakage of contrast I peritoneal cavity

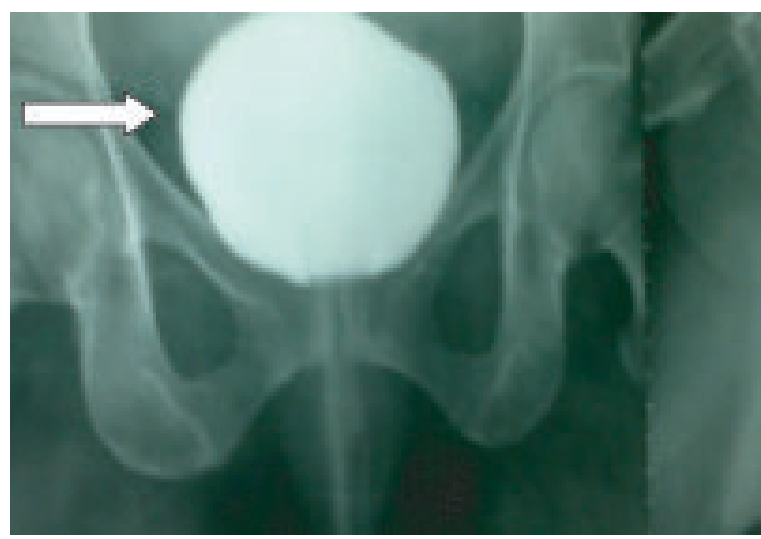

Fig.-3 : Follow up cystogram showing bladder with normal contour

\section{Discussion}

Injury to adjacent structures is a recognized complication during gynaecological procedures including hysterectomy and laparoscopic surgery and urinary bladder is the most common organ injured during such procedures ${ }^{7-10}$. Presence of adhesions from previous surgery or intra-abdominal disease such as endometriosis or pelvic inflammatory disease predisposes the patient to such injury.

Signs indicating bladder injury intraoperatively are bloody urine and the presence of gaseous distention of the urine bag indicative of a communication between the bladder and the intraperitoneal space (the catheter bag sign) and the injury may be confirmed by instillation of methylene blue in the bladder ${ }^{11}$.

The bladder injury may be recognized and repaired surgically intra-operatively but if the wound is less than 
$1 \mathrm{~cm}$ it can be treated conservatively by continuous drainage with Foley's catheter for about a week allowing spontaneous healing of the wound to occur ${ }^{12}$.

If bladder injury is missed intraoperatively, the patient may present with voiding difficulty, oliguria, haematuria, pyrexia, abdominal distension, nausea and vomiting and peritonitis secondary to the formation of urinoma as early as 1 to 2 days after the incident ${ }^{13}$.

There was no clue to raise suspicion of bladder injury in our patient as the surgery and the early postoperative period was reported as uneventful.

Retrospectively, the presentation of our patient with features of urinary infection seven months after hysterectomy may be linked with bladder catheterization which is often done during any pelvic surgery which was perhaps controlled with the antibiotics she received at that time but recurred later.

In most patients with urinary ascites, there may be great disturbances in serum biochemistry mimicking renal failure such as elevation in the urea and creatinine as well as hyperkalaemia and hyponatraemia ${ }^{14}$. This occurs because urine has higher concentration of urea, creatinine and potassium than serum resulting in diffusion of these substances into the lower concentration gradient, while sodium diffuses in the opposite direction ${ }^{15}$.

In our patient the serum urea, and creatinine were markedly raised but this was attributed to NSAID induced AKI as these declined significantly with stoppage of NSAID without any renal replacement therapy. This improvement in renal function could have partly contributed to the delay in diagnosis of bladder injury in our patient.

In this case the clue to the diagnosis was the markedly elevated ascitic fluid urea and creatinine levels despite near normal serum urea and creatinine. The persistence of oliguria and rapid reaccumulation of ascites in the face of continued improvement in the biochemical parameters of renal function to near normal values led us to strongly suspect a communication between the urinary tract and the peritoneal cavity.

\section{Conclusion}

A high index of suspicion of bladder injury following pelvic surgery should be present in patients with illdefined symptoms even if no expected typical biochemical changes in blood are seen and the suspicion can be supported by finding raised urea and creatinine in ascitic fluid.

The diagnosis of inadvertent bladder injury may be delayed due to the insidious development of non-specific symptoms.

\section{References}

1. Nikhil Vasdev, Malcolm G. Coulthard, Mike N. De la hunt, Bosena Starzyk, Milos Ognjanovic, Willetts IE. Neonatal urinary ascites secondary to urinary bladdar rupture. Journal of Pediatric Urology 2009 ; 5 : 100-104.

2. Wasnick R J. Neonatal urinary ascites secondary to ureteropelvic junction obstruction.Urology 1987;30 : 470-71.

3. Tank E S, Carey T C , Seifert A L.Management of neonatal urinary ascites. Urology 1987; 16 : 270-73.

4. Ismail Hassan, Carolina López, Harry Gee, Philip ToozsHobson.Urinary ascites following caesarean section: An unusual presentation of bladder injury. European Journal of Obstetrics \& Gynecology and Reproductive Biology 2009;147(2): 237-38.

5. Khan MA, Zargar SA, Javid G, Khan BA, Shah AH, Gulzar GM, Sodhi JS.Vesicoperitoneal fistula - an unusual cause of tense ascites. Indian J Gastroenterol 2007 ;26(3):138-39.

6. Bourdeau GV, Jindal SL, Gillies RR, Berry JV.Urinary ascites secondary to ureteroperitoneal fistula. Urology. 1974 Aug;4(2):209-11.

7. M. Schapira, H. Dizerens, A. Essinger, J. -P. Wauters, P. Loup, W. Von Niederhausern. Urinary Ascites After Gynaecological Laparoscopy The Lancet 1978; Volume 311 (8069):871-72.

8. J. Chandler Williams, John A. Heaney, William Young.Respiratory distress following cesarean section: Cryptic presentation of bladder injury. Urology September 1994; 44( 3): 441-43.

9. Ostrzenski A, Ostrzenska KM. Bladder injury during laparoscopic surgery. Obstet Gynecol Surv 1998;53:175-80

10. Wiwanitkit, V. Laparoscopic bladder injury and ascites. Journal of Minimal Access Surgery. 2011; 7(2): 162.

11. Ho AM, Roth P, Cowan WD. Gaseous distension of the urinary bag indicating bladder perforation during laparoscopic pelvic procedures. Int J Gynecol Obstet 1996;55:297-98.

12. Al-Mandeel H, Qassem A. Urinary ascites secondary to delayed diagnosis of laparoscopic bladder injury. J Min Access Surg 2010;6:50-52.

13. Nachshen S, Abusaid D, Nauta M, Govind A, Yoong W. The "catheter bag" sign revisited: A simple way detecting bladder truma during operative laparoscopic procedures. Arch Gynaecol Obstet 2008;277:65-66.

14. Tai CK, Li SK, Hou SM, Fan CW. Bladder injury mimicking acute renal failure after cesarean section. Surg Laparosc Endosc Percutan Tech 2008;18:301-303.

15. Vilos GA, Haebe J, Crumley TL, Maruncic MA, King JH, Denstedt JD. Serum biochemical changes after laparoscopy may be indicators of bladder injury. J Am Assoc Gynecol Laparosc 2001;8:285-90. 